\title{
Influence of Intersubband Scattering on the Magnetic Field Dependence of the Conductivity Tensor
}

\author{
J. Siwiec-Matuszyk*, M. Baj, A. Babiński \\ Institute of Experimental Physics, Warsaw University \\ Hoża 69, 00-681 Warsaw, Poland \\ AND J. KASPRZAK \\ Laboratoire de Spectrométrie Physique, Université J. Fourier - Grenoble \\ 38402 Saint Martin d'Hères Cedex, France \\ In this paper we show that intersubband scattering can lead to apparent \\ inconsistency of the experimental results obtained by means of classical and \\ quantum transport measurements and this discrepancy is entirely connected \\ with the usage of classical formulae to describe magnetic field dependence of \\ a conductivity tensor. We prove that there is no contradiction in our observa- \\ tions and that the models describing quantum oscillations and magnetic-field \\ dependence of the conductivity tensor, which are present in the literature, \\ complement each other.
}

PACS numbers: 72.20.My, 73.50.Jt, 73.21.Fg, 73.61.Ey

\section{Introduction}

Shubnikov-de Haas (SdH) effect is a powerful method used to characterize two-dimensional electron gas (2DEG) in semiconductor heterostructures [1-4]. For 2DEG with only one subband populated, SdH oscillations have a simple mathematical form [1]. However, for various semiconductor devices high density 2DEG is required and the increase in dopant concentration leads to a second subband occupation. In these cases we usually expect $\mathrm{SdH}$ oscillations originating from all the occupied subbands, but it is also possible that additional intersubband effects occur.

\footnotetext{
*corresponding author; e-mail: siwiec@fuw.edu.pl
} 
It was shown $[5,6]$ that one-subband model of resistivity oscillations can be easily extended to the case of two occupied subbands. Then the resistivity tensor is composed of four components with different frequencies and different temperature dependences. Two of them are standard SdH oscillations proportional to the density of state oscillations for both occupied subbands. The third component, with the frequency proportional to the total electron concentration, contains temperature damping factor $2 X / \sinh (2 X)$ (where $X=2 \pi^{2} k_{\mathrm{B}} T / \hbar \omega_{\mathrm{c}}$ ), which is stronger than that for $\mathrm{SdH}$ oscillations, and thus this component does not contribute to the observed oscillations, especially at higher temperature. The last one does not depend on temperature and has the frequency proportional to the difference of both concentrations. Moreover, the phase of these oscillations is opposite to the phase of $\mathrm{SdH}$ oscillations. This component was termed magnetointersubband (MIS) oscillations and is responsible for the observed beating and a phase change in magnetic field $[3,7,8]$ which is visible especially when the electron density of the second subband is small, in other words $n_{1} \approx n_{1}-n_{2}$. It is important that MIS oscillations disappear rapidly when the electron quantum lifetime of any subband is small.

The appearance of intersubband scattering raises some doubts concerning the use of a common assumption of an additive form of a conductivity tensor in the analysis of an experiment. Studenikin et al. [9] showed the mathematical model of a conductivity tensor with two occupied subbands and intersubband scattering. Solution of the Boltzmann equation led to the following formulae:

$$
\sigma_{x x}=\sum_{\alpha=1}^{2} a_{\alpha} n_{\alpha} \frac{e^{2}}{m^{*}}, \quad \sigma_{x y}=\sum_{\alpha=1}^{2} b_{\alpha} n_{\alpha} \omega_{\mathrm{c}} \frac{e^{2}}{m^{*}},
$$

where each coefficient $a_{\alpha}$ and $b_{\alpha}$ depends on magnetic field, mobilities, and concentrations of both subbands as well as probabilities of intersubbands scattering. So, it is not possible to separate contributions of each subband because all the parameters are mixed. Thus the use of common formulae leads to differences between the values of parameters obtained from the fitting of a conductivity tensor and the analysis of quantum oscillations.

In this paper we show that such a discrepancy can by really significant. In our results the difference is not only in the values obtained by means of different methods, but the results seem to contradict and exclude each other. The aim of this paper is to explain this apparent contradiction basing on a thorough analysis of existing models. We show that these results really do not exclude but rather complement each other.

\section{Experimental results}

The object of the research was the modulation-doped AlGaAs/InGaAs/ GaAs heterostructure. We have measured resistivity tensor at $4 \mathrm{~K}$ in a magnetic field up to 7 T. Persistent photoconductivity effect was used to change electron 
density from $1.09 \times 10^{12} \mathrm{~cm}^{-2}$ to $1.58 \times 10^{12} \mathrm{~cm}^{-2}$. The sample was illuminated by LED.

The analysis of the $\rho_{x x}$ component of the resistivity tensor shows that additional magnetooscillations appear in a small magnetic field for electron density exceeding $n \approx 1.28 \times 10^{12} \mathrm{~cm}^{-2}$. As it is shown in Fig. 1 , for three different values of $n_{\mathrm{SdH}}$, at low magnetic field a node appears and the phase of oscillations is modified. Our analysis shows that the frequency of oscillation also changes a little at

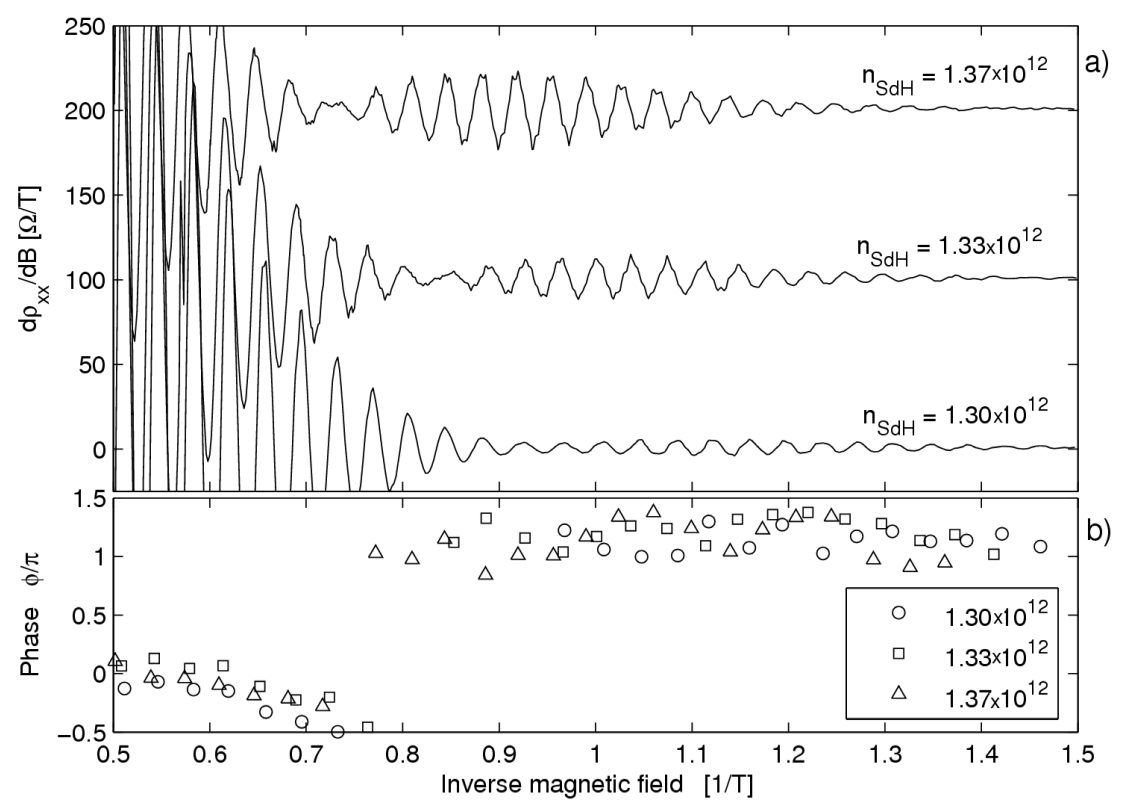

Fig. 1. The first derivative of magnetoresistance $\rho_{x x}$ upon magnetic field for three different values of $n_{\mathrm{SdH}}$ (a) and residual phase (see [6]) of oscillations (b) as a function of inverse magnetic field. At low magnetic field the node is clearly seen. At the same time the phase of oscillations changes.

the same time. Since these properties are a fingerprint of MIS oscillations, we can relate the low-field frequency of oscillations to $n_{1}-n_{2}$ and hence calculate the value of the electron density of the second subband, $n_{2}$. It is very important to notice that the observation of MIS oscillations implies that the electron quantum lifetime of the second subband cannot be considerably smaller than the quantum lifetime of the first one. However, the fitting of the magnetic-field dependence of the nonoscillatory part of the conductivity tensor component $\sigma_{x x}$ does not reveal any other conducting channel with mobility comparable to the mobility of electrons on the first subband, $\mu_{1}$ (see Fig. 2). The second conductivity channel has a very low mobility - in most of the fits we even used a model with one subband only and a constant background (open points in Figs. 2 and 4). Figure 3 presents the low field Hall concentration versus the first-subband concentration obtained from 


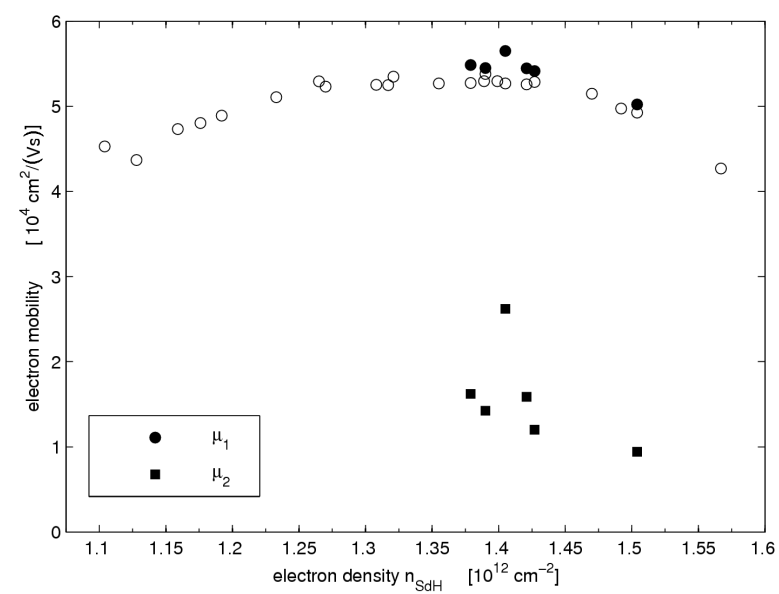

Fig. 2. Electron mobility for the first (circles) and the second (squares) subbands, $\mu_{1}$ and $\mu_{2}$, respectively, obtained from the fitting basing on the formulae typically used for the case of independent conduction channels, drawn versus the concentration of the first subband, $n_{\mathrm{SdH}}$. In most of the fits we used a model with one subband only and a constant background (open points). We can see the maximum of $\mu_{1}$ near $n_{\mathrm{SdH}} \approx 1.3 \times 10^{12} \mathrm{~cm}^{-2}$ connected with turning on the intersubband scattering.

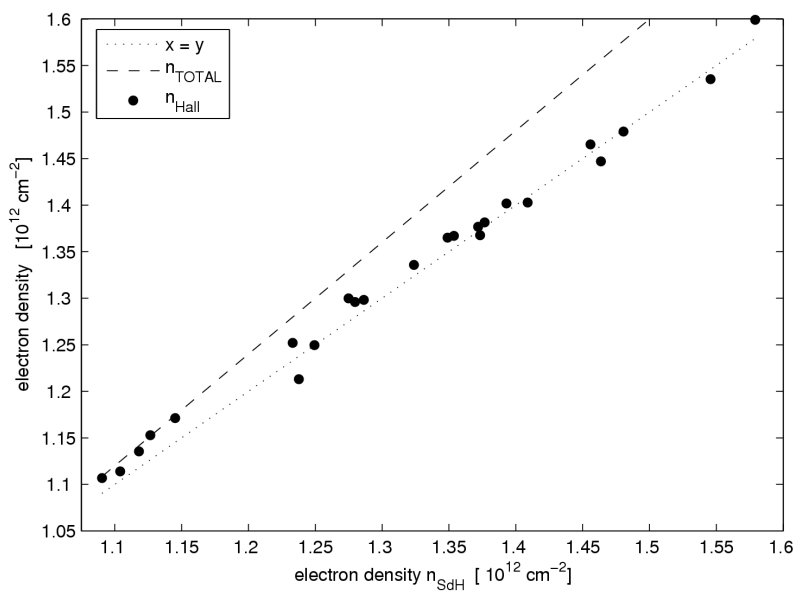

Fig. 3. Hall concentration $n_{\text {Hall }}$ (dark circles) as a function of the concentration of the first subband, $n_{\mathrm{SdH}}$. The slope of this dependence is nearly one (dotted line). Dashed line shows the total concentration $n_{1}+n_{2}$ which would correspond to the Hall concentration if both subbands had the same mobility.

SdH oscillations, $n_{\mathrm{SdH}}$. As we can see, the slope of this dependence (dark points) is nearly one (dotted line), whereas the second subband with mobility comparable to $\mu_{1}$ should increase this slope (dashed line). On the other hand, $\mu_{1}$ dependence 


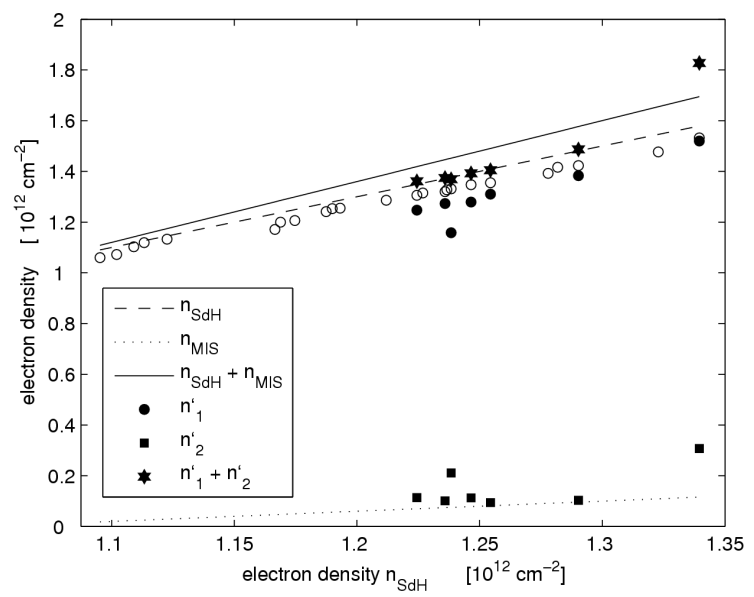

Fig. 4. Values of electron concentration for both observed subbands obtained from different methods. Lines show the results of the analysis of magnetooscillations. Points show the results of the fitting of the nonoscillatory part of the conductivity tensor. In most of the fits we used a model with one subband only and a constant background (open points).

on $n_{\mathrm{SdH}}$ is typical of the case of gradual turning on the intersubband scattering $[3,7,10]$ (see Fig. 2).

So it follows that the results of the analysis of quantum oscillations and magnetic-field dependence of a nonoscillatory part of $\sigma_{x x}$ conductivity tensor component seem to contradict each other:

1. They lead to different values of electron concentrations of both subbands (Fig. 4).

2. The values of quantum lifetime $\tau_{\mathrm{q}}$ and transport scattering time $\tau_{\mathrm{tr}}$ exhibit even a more serious problem. Low mobility of the second subband implies the low value of $\tau_{\operatorname{tr} 2}$ and, as a consequence, the low value of $\tau_{\mathrm{q} 2}$. But the low value of $\tau_{\mathrm{q} 2}$ should cause a very rapid damping of the component which describes MIS oscillations, so these oscillations should not have been observed.

\section{Discussion}

Our detailed analysis of the model presented in the work [9] (the details of the calculation will be published elsewhere) has shown that the complicated and indirect magnetic-field dependence of $\sigma_{x x}$ and $\sigma_{x y}$ given by formulae (1.1) can be expressed in a simple form as follows:

$$
\sigma_{x x}=\sum_{i=1}^{2} \frac{e n_{1}^{\prime} \mu_{i}^{\prime}}{1+\left(\mu_{i}^{\prime} B\right)^{2}} \quad \sigma_{x y}=\sum_{i=1}^{2} \frac{e n_{1}^{\prime}\left(\mu_{i}^{\prime}\right)^{2} B}{1+\left(\mu_{i}^{\prime} B\right)^{2}} .
$$

This form is similar to a commonly used one with additive contribution of various 
conduction channels. The magnetic field dependence is the same but the coefficients $n_{i}^{\prime}$ and $\mu_{i}^{\prime}$ are not related to any definite subband. They depend on all the parameters of 2DEG just like $a_{\alpha}$ and $b_{\alpha}$ coefficients in formulae (1.1). So while it is possible to use formulae (3.1) for the analysis, the values of electron densities obtained from the fitting can differ considerably from those obtained from the analysis of the magnetooscillations. However, we have shown that the relation $n_{1}+n_{2}=n_{1}^{\prime}+n_{2}^{\prime}$ is valid - this can be used to reduce a number of fitting parameters, on condition that it is possible to obtain the values of $n_{1}$ or $n_{2}$ from the analysis of oscillations. Moreover, the parameters $\mu_{i}^{\prime}$ are not proportional to the intrasubband scattering times $\tau_{\mathrm{tr}, i}$.

It is interesting to note that using formulae (3.1) the relation $\mu_{1}^{\prime}>\mu_{2}^{\prime}$ (subscript "1" corresponds to the conduction channel with higher concentration, i.e. mainly related to the first subband) can be obtained from the analysis of experimental data also for the case when in reality $\tau_{\mathrm{tr}, 1} \approx \tau_{\mathrm{tr}, 2}$ or even $\tau_{\mathrm{tr}, 1}<\tau_{\mathrm{tr}, 2}$ !

It means that in the case of intersubband scattering the use of formulae (3.1) for the analysis of experimental data can lead to completely erroneous conclusions. This is illustrated in Fig. 5, which shows the results of the calculations of the ratio $\mu_{1}^{\prime} / \mu_{2}^{\prime}$ versus $f$ for $\tau_{\mathrm{tr}, 1}=\tau_{\mathrm{tr}, 2}$ (solid line). The factor $f=\widetilde{\nu} / \nu_{12}$ is connected

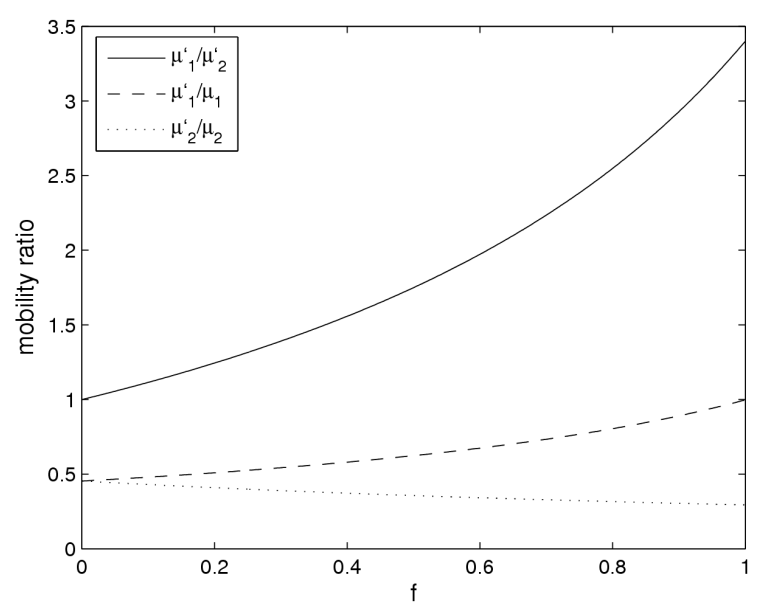

Fig. 5. The calculated ratios $\mu_{1}^{\prime} / \mu_{2}^{\prime}$ (solid line), $\mu_{1}^{\prime} / \mu_{1}$ (dashed line) and $\mu_{2}^{\prime} / \mu_{2}$ (dotted line) as a function of $f$ for $\mu_{1}=\mu_{2}$. For this calculation we used the model described in the work [9] and the values $n_{1}=1.25 \times 10^{12} \mathrm{~cm}^{-2}, n_{2}=2.02 \times 10^{11} \mathrm{~cm}^{-2}, \mu_{1}=\mu_{2}=$ $50000 \mathrm{~cm}^{2} /(\mathrm{Vs})$ and $\nu_{12}=1.2 / \nu_{1}$ where $\nu_{1} \sim 1 / \tau_{\text {tr1 }}$. This shows that the ratio $\mu_{1}^{\prime} / \mu_{2}^{\prime}$ can be considerably higher than the one even for $\tau_{\mathrm{tr}, 1}=\tau_{\mathrm{tr}, 2}$.

to intersubband scattering probabilities: $\widetilde{\nu}=\sum_{\boldsymbol{k}} W\left(1 \boldsymbol{k}, 2 \boldsymbol{k}^{\prime}\right) \cos (\theta)$ and $\nu_{12}=$ $\sum_{\boldsymbol{k}^{\prime}} W\left(1 \boldsymbol{k}, 2 \boldsymbol{k}^{\prime}\right)$, where $\theta$ is a scattering angle - see the work [9]). It is visible that even for $\tau_{\mathrm{tr}, 1}=\tau_{\mathrm{tr}, 2}$ the presence of intersubband scattering leads to the value of $\mu_{1}^{\prime} / \mu_{2}^{\prime}$ which is considerably higher than one. The parameter $f$ can be a 
measure of anisotropy of scattering angle (by analogy to $\tau_{\mathrm{q}} / \tau_{\mathrm{tr}}$ ratio [11]). As we can see in Fig. 5, a high value of $\mu_{1}^{\prime} / \mu_{2}^{\prime}$ occurs when $f$ is close to one, i.e. for a small-angle scattering mechanism dominant.

\section{Conclusion}

In this paper we studied classical and quantum magnetotransport in AlGaAs/InGaAs/GaAs heterostructure with two occupied subbands. We have shown that models describing quantum oscillations and magnetic-field dependence of the conductivity tensor, which are present in the literature, complement each other. A thorough analysis has shown that:

- In the case of 2DEG with two occupied subbands attendance of intersubband scattering does not change the form of the magnetic-field dependence of the conductivity tensor.

- Complicated formulae of the magnetic-field dependence of the conductivity tensor with intersubband scattering can be converted to a form, which is identical to the case of independent parallel conduction channels.

- The fitting of formulae (3.1) to the experimental data can lead to the values of $n_{1}^{\prime}, n_{2}^{\prime}$ and $\mu_{1}^{\prime}, \mu_{2}^{\prime}$ very much different from the real concentrations and mobilities. In particular:

1. The values of $n_{1}^{\prime}$ and $n_{2}^{\prime}$ obtained from this fitting can differ considerably from the concentrations occupying both subbands evaluated from the analysis of the magnetooscillations.

2. The ratio $\mu_{1}^{\prime} / \mu_{2}^{\prime}$ obtained from this fitting can by substantially different from $\tau_{\mathrm{q}, 1} / \tau_{\mathrm{q}, 2}$.

This permits us to explain apparent discrepancy between the experimental results of quantum and classical transport presented in this paper.

\section{Acknowledgments}

This work has been supported by the Ministry of Science and Higher Education (Poland) under grant No. 2 PO3B 02023.

\section{References}

[1] A. Isihara, L. Smrcka, J. Phys. C 19, 6777 (1986).

[2] P.T. Coleridge, R. Stoner, R. Fletcher, Phys. Rev. B 39, 1120 (1989).

[3] R. Fletcher, E. Zaremba, M. D'Idorio, C.T. Foxon, J.J. Harris, Phys. Rev. B 41, 10649 (1990).

[4] P.T. Coleridge, Phys. Rev. B 44, 8126 (1991).

[5] P.T. Coleridge, Semicond. Sci. Technol. 5, 961 (1990). 
[6] T.H. Sander, S.N. Holmes, J.J. Harris, D.K. Maude, J.C. Portal, Phys. Rev. B 58, 13856 (1998).

[7] H.L. Störmer, A.C. Gossard, W. Wiegmann, Sollid State Commun. 41, 707 (1982).

[8] S. Mori, T. Ando, Phys. Rev. B 19, 6433 (1979).

[9] S.A. Studenikin, A.V. Chaplik, I.A. Panaev, G. Salis, K. Ensslin, K. Maranowski, A.C. Gossard, Semicond. Sci. Technol. 14, 604 (1999).

[10] L.R. Gonzales, J. Krupski, T. Szwacka, Phys. Rev. B 54, 7658 (1996).

[11] J.P. Harrang, R.J. Higgins, R.K. Goodall, P.R. Jay, M. Laviron, P. Delescuse, Phys. Rev. B 32, 8126 (1985). 